\title{
Curcumin induces apoptotic cell death and protective autophagy in human gastric cancer cells
}

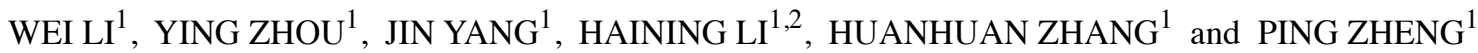 \\ ${ }^{1}$ Center of Research Laboratory, The First People's Hospital of Lianyungang; \\ ${ }^{2}$ Department of Clinical Laboratory Diagnostics, Kangda College of Nanjing Medical University, \\ Lianyungang, Jiangsu 222001, P.R. China
}

Received October 22, 2016; Accepted February 2, 2017

DOI: $10.3892 / o r .2017 .5637$

\begin{abstract}
Curcumin possesses an anticancer effect against a wide assortment of tumors with selective cytotoxicity for tumor cells. However, the mechanism involved in the curcumin-induced anticancer effect remain unclear. In the present study, we investigated the efficacy of curcumin against human gastric cancer cell growth and the molecular mechanism involved. Our results demonstrated that curcumin inhibited the viabilities of gastric cancer cell lines BGC-823, SGC-7901 and MKN-28 in both a time- and dose-dependent manner. In addition, curcumin treatment induced gastric cancer cell apoptosis in a dose-responsive manner. Western blotting of apoptosis-related proteins further confirmed the pro-apoptotic potential of curcumin. After exposure to curcumin, a robust induction of autophagy was observed in gastric cancer cells, which was characterized by the formation of acidic vesicular organelles (AVOs), conversion of LC3-I to LC3-II and an increase in the levels of autophagy-related proteins. Activation of the PI3K/Akt/mTOR signaling pathway was suppressed in gastric cancer cells with curcumin treatment. However, administration of the autophagy inhibitor 3-methyladenine (3-MA) significantly promoted the apoptotic cell death induced by curcumin. Collectively, our findings provide new evidence that curcumin induces apoptotic cell death and protective autophagy in human gastric cancer cells in vitro. Autophagy inhibitor treatment may provide a novel and effective strategy for improving the anticancer effect of curcumin against gastric cancer.
\end{abstract}

\section{Introduction}

Gastric cancer is one of the most common malignant tumors worldwide (1). Although improvements in the early diagnosis

Correspondence to: Dr Wei Li, Center of Research Laboratory, The First People's Hospital of Lianyungang, 182 Tongguan Road, Lianyungang, Jiangsu 222001, P.R. China

E-mail: medical112@126.com

Key words: curcumin, gastric cancer, autophagy, apoptosis and treatment have recently been made, the morbidity and mortality rates are still high (2). For patients with advanced gastric cancer, chemotherapy is still the primary therapeutic regimen as preoperative and postoperative adjuvant therapy $(3,4)$.

Curcumin, a polyphenol extracted from turmeric (Curcuma longa), is an agent with anticancer potential against various types of tumors. As a natural compound, it has been proven to be effective with minimal toxicity, which selectively acts on cancer cells over normal cells $(5,6)$. Hitherto, curcumin and its analog have been reported to play an anticancer role in several tumor models, including glioblastoma (7), liver (8), colorectal (9), lung (10), ovarian (11), breast (12) and oral (13) cancer. The underlying mechanisms have been demonstrated to be associated with the inhibition of proliferation, angiogenesis, invasion and metastasis of cancer cells, or apoptosis induction by curcumin (14-16). However, research on the anticancer effect of curcumin against gastric cancer and the related molecular mechanism remain to be elucidated.

Autophagy, a self-degradative process that is highly conserved among different types of mammalian cells, has been demonstrated in studies to play a critical, and complicated role in cancer development and progression as well as therapy resistance (17-19). Autophagy is an intracellular process starting with the formation of double membrane vesicles. Then, the contents of the vesicles, long-lived proteins and damaged organelles, are delivered to the lysosome to degrade. It has been found that autophagy exerts a dual effect on cancer $(17,18)$. On one hand, autophagy may function as a tumor suppressor by preserving cellular integrity. Autophagy-deficient cells are prone to develop tumorigenesis in vivo. On the other hand, autophagy may play an oncogenic role by maintaining tumor cell survival and preventing apoptotic cell death after anticancer treatment. Recently, several studies demonstrated that natural compounds with anticancer abilities could induce autophagy in different types of tumors (20-22). However, the precise role of autophagy (tumor suppressor or promoter) involved in the mechanism of natural compound-induced cancer cell death needs to be further studied.

In the present study, the anticancer effect of curcumin on human gastric cancer and the underlying mechanism were investigated. We designed the study to determine the regulatory role of curcumin in the survival or apoptosis of 
three different gastric cancer cell lines BGC-823, SGC-7901 and MKN-28. Thereafter, the effect of curcumin-induced autophagy on gastric cancer cells and the related molecular mechanism were further elucidated.

\section{Materials and methods}

Cell culture and reagents. Human gastric cancer cell lines BGC-823, SGC-7901 and MKN-28 were gifts from The First Affiliated Hospital of Soochow University (Jiangsu, China). The cells were routinely cultured in RPMI-1640 medium supplemented with $10 \%$ (v/v) fetal bovine serum (FBS) (both from Gibco, Invitrogen Corporation, Carlsbad, CA, USA) at $37^{\circ} \mathrm{C}$ in a humidified atmosphere of $5 \% \mathrm{CO}_{2}$. The adherent cells were subcultured every 2-3 days and harvested for the subsequent experiments.

Reagents of curcumin (C1386), MTT (M2128), acridine orange (AO; A6014), 3-methyladenine (3-MA; M9281) and dimethyl sulfoxide (DMSO; D2650) used in the present study, were all obtained from Sigma-Aldrich (St. Louis, Mo, USA). Curcumin or autophagy inhibitor 3-MA was dissolved in DMSO as stock solution and kept at $-20^{\circ} \mathrm{C}$ prior to usage. In addition, MTT or AO was dissolved in phosphate-buffered saline (PBS) for solution preparation.

Cell viability assay. The regulatory effect of curcumin on gastric cancer cell growth was evaluated using an MTT assay. Briefly, cells were seeded at a density of $1 \times 10^{4}$ cells/well in a 96-well culture plate, incubated overnight and treated with or without increasing amounts of curcumin $(0-200 \mu \mathrm{M})$ for 24 , 48 and $72 \mathrm{~h}$, respectively. Subsequently, $10 \mu \mathrm{l}$ of MTT solution $(5 \mathrm{mg} / \mathrm{ml})$ was added into each well and incubated with the cells at $37^{\circ} \mathrm{C}$ for another $4 \mathrm{~h}$. Then, the solution was discarded and $150 \mu \mathrm{l}$ of DMSO was added into the remaining cells to dissolve the formazan crystals. The optical density (OD) at $490 \mathrm{~nm}$ was measured using a microplate reader (Benchmark, Bio-Rad Laboratories, Hercules, CA, USA). The formula used in the present study was: Cell viability $(\%)=(\mathrm{OD}$ of the experimental sample/OD of the control group) $\mathrm{x} 100 \%$.

TUNEL assay. TUNEL staining was performed in the present study to determine the apoptosis-promoting effect of curcumin on gastric cancer cells. In brief, the cells were seeded in a 24-well plate at a density of $7 \times 10^{4}$ cells/well. After incubation overnight, gastric cancer cells were exposed to the indicated concentrations of curcumin for $48 \mathrm{~h}$. Then, the cells were fixed with $4 \%$ paraformaldehyde and stained for the TUNEL assay. In the present study, TUNEL staining was performed with a One Step TUNEL Apoptosis Assay kit (Beyotime Biotechnology, Nantong, China) according to the manufacturer's instructions. Thereafter, the cells were observed and photographed under a fluorescence microscope (Olympus Optical Co., Hamburg, Germany) and the red stained nuclei were considered as TUNEL-positive.

Flow cytometric analysis. Flow cytometry was used to confirm the apoptosis-inducing effect of curcumin on gastric cancer cells. After $12 \mathrm{~h}$ of incubation, gastric cancer cells seeded into a 6 -well plate $\left(3 \times 10^{5}\right.$ cells/well) were treated with the indicated amounts of curcumin for $48 \mathrm{~h}$. Then, the cells were harvested, washed with ice-cold PBS and re-suspended with the complete culture medium. The cell suspension was incubated with a Muse ${ }^{\mathrm{TM}}$ Annexin V \& Dead Cell Assay kit (EMD Millipore, Hayward, CA, USA) according to the manufacturer's instructions, and analyzed on a Muse ${ }^{\mathrm{TM}}$ Cell Analyzer system (EMD Millipore). Data were analyzed using Muse $^{\mathrm{TM}}$ Analysis software (EMD Millipore).

Western blotting. A western blotting assay was performed to evaluate the expression of apoptosis- or autophagy-related proteins in human gastric cancer cells. After exposure to the indicated amounts of curcumin for $48 \mathrm{~h}$, the cells were harvested and solubilized in cold RIPA buffer supplemented with complete protease inhibitors. Equal amounts of cellular lysates were separated on SDS-PAGE and electrophoretically transferred to polyvinylidine fluoride (PVDF) membranes. The membranes were then blocked with $5 \%(\mathrm{w} / \mathrm{v})$ blotting grade milk for $1 \mathrm{~h}$ and then incubated with the primary antibodies overnight at $4^{\circ} \mathrm{C}$. The primary antibodies anti-Bcl-2, anti-Bax, anti-caspase-3 and anti-caspase- 9 were purchased from Merck-Millipore (Billerica, MA, USA); anti-LC3 was obtained from Abcam (Cambridge, MA, USA); anti-Beclin1 and anti-Atg7 were purchased from Abgent (San Diego, CA, USA); anti-Atg12-Atg5, anti-p-Akt, anti-Akt, anti-p-mTOR, anti-mTOR, anti-p-p70S6K and anti-p70S6K were obtained from Cell Signaling Technology (Beverly, MA, USA). Anti$\beta$-actin (Santa Cruz Biotechnology, Santa Cruz, CA, USA) was used as an internal control. Subsequently, the membranes were washed and treated with the appropriate HRP-conjugated secondary antibodies for $2 \mathrm{~h}$, and the bound antibodies were visualized by an enhanced chemiluminescence (ECL) reagent (Beyotime Biotechnology).

Detection of acidic vesicular organelles (AVOs). Acridine orange (AO) staining was used to determine the vacuolar acidification of autophagosomes, which is a characteristic of efficient autophagy. Briefly, gastric cancer cells were seeded in a 6-well plate at a density of $3 \times 10^{5}$ cells/well and incubated overnight. Following exposure to the indicated concentrations of curcumin for $48 \mathrm{~h}$, the cells were treated with $\mathrm{AO}(1 \mu \mathrm{g} / \mathrm{ml})$ for $15 \mathrm{~min}$, washed with PBS and visualized under a fluorescence microscope (Olympus Optical Co.). The orange-red fluorescence in the cytoplasm was recognized as AO-positive staining.

Statistical analysis. All quantitative data are expressed as the mean \pm SEM and plotted with the GraphPad Prism software 6.0 (GraphPad Software, La Jolla, CA, USA). Statistical analysis was performed by non-parametric Mann-Whitney U test using SPSS 16.0 statistical software and a P-value $<0.05$ was considered as statistically significant.

\section{Results}

Gastric cancer cell proliferation is regulated by curcumin. To confirm the potential role of curcumin in gastric cancer cell growth, we performed an MTT assay to assess the cell viability after treatment with curcumin over a wide range of concentrations $(0-200 \mu \mathrm{M})$. As shown in Fig. 1A, curcumin inhibited the proliferation of three different gastric cancer 
A

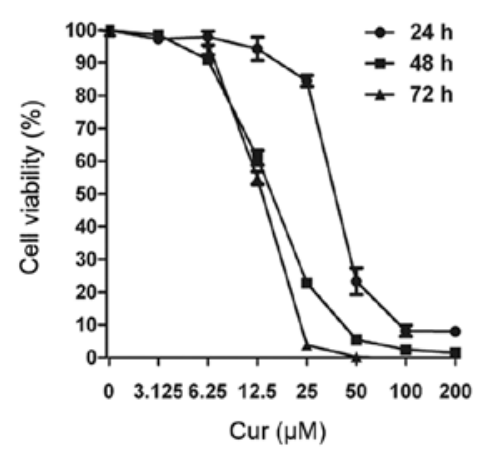

B

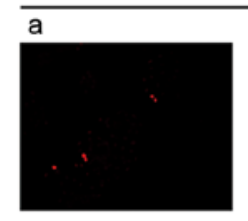

c

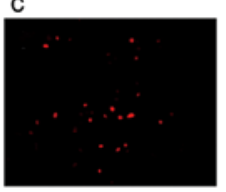

BGC-823

GC-823

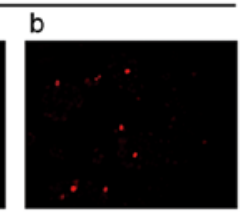

d

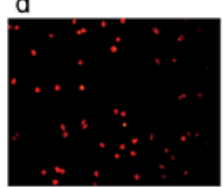

SGC-7901

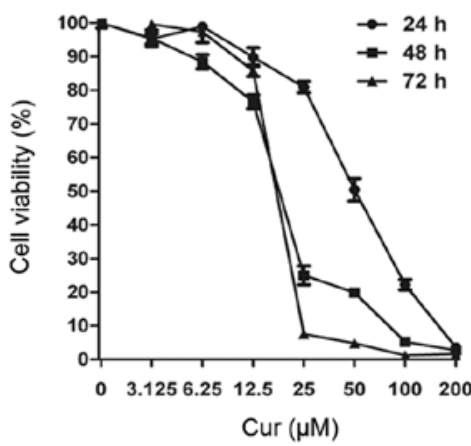

SGC-7901
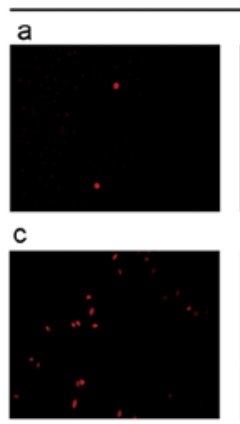

MKN-28

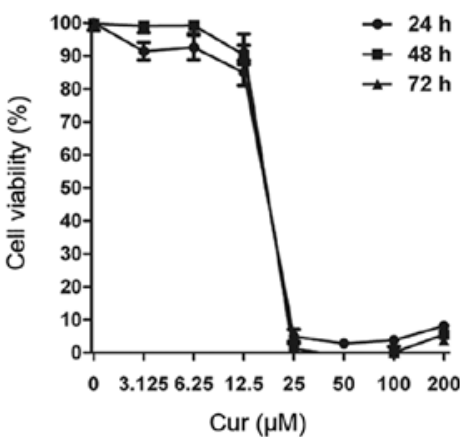

MKN-28

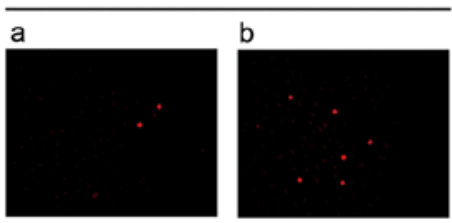

c

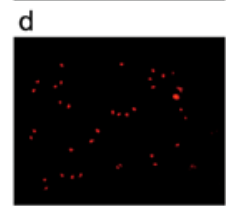

Figure 1. Viability and apoptosis in human gastric cancer cells after curcumin treatment. (A) BGC-823, SGC-7901 and MKN-28 cells were treated with curcumin at the indicated concentrations for 24,48 and $72 \mathrm{~h}$, and cell viability was assessed using an MTT assay. (B) BGC-823, SGC-7901 and MKN-28 cells were treated with curcumin at increasing concentrations for $48 \mathrm{~h}$ and apoptotic cell death was determined by TUNEL staining (magnification, $\mathrm{x} 200$ ). BGC-823: a, DMSO; b, $5 \mu \mathrm{M}$ curcumin; c, $10 \mu \mathrm{M}$ curcumin; d, $15 \mu \mathrm{M}$ curcumin. SGC-7901 and MKN-28: a, DMSO; b, $5 \mu \mathrm{M}$ curcumin; c, $10 \mu \mathrm{M}$ curcumin; $\mathrm{d}, 20 \mu \mathrm{M}$ curcumin. Cur, curcumin.

Table I. The $\mathrm{IC}_{50}$ values $(\mu \mathrm{M})$ of curcumin against gastric cancer cells.

\begin{tabular}{lccc}
\hline Cell lines & $24 \mathrm{~h}$ & $48 \mathrm{~h}$ & $72 \mathrm{~h}$ \\
\hline BGC-823 & 37.58 & 15.18 & 13.00 \\
SGC-7901 & 50.45 & 18.53 & 16.72 \\
MKN-28 & 16.17 & 15.84 & 14.04 \\
\hline
\end{tabular}

cell lines BGC-823, SGC-7901 and MKN-28 in both a time- and dose-dependent manner, albeit to varying degrees. The $\mathrm{IC}_{50}$ values of curcumin against gastric cancer cells are presented in Table I. After $24 \mathrm{~h}$ of culture, MKN-28 cells were found to be the most sensitive to curcumin $\left(\mathrm{IC}_{50}=16.17 \mu \mathrm{M}\right)$ and SGC-7901 cells were the most resistant $\left(\mathrm{IC}_{50}=50.45 \mu \mathrm{M}\right)$, whereas BGC-823 cells provided an intermediate sensitivity $\left(\mathrm{IC}_{50}=37.58 \mu \mathrm{M}\right)$. At $48 \mathrm{~h}$ after treatment, the $\mathrm{IC}_{50}$ values of each cell line decreased and were similar. Thus, the regulatory effects of curcumin on gastric cancer cells were all observed after $48 \mathrm{~h}$ of exposure in our following studies, at a range of concentrations between 0 and $20 \mu \mathrm{M}$.

Apoptotic cell death in gastric cancer cells is induced by curcumin. To explore the effect of curcumin on gastric cancer cell apoptosis, a combination of TUNEL staining, flow cytometry and western blotting assays was performed in our investigation. As shown in Fig. 1B, the nuclei of curcumin-treated gastric cancer cells were condensed and exhibited bright red fluorescence at $48 \mathrm{~h}$ post-treatment in a dose-dependent manner. Consistent with this observation, the results of flow cytometric analysis indicated a significant increase in the apoptotic population (early apoptosis plus late apoptosis) of gastric cancer cells treated with the increasing concentrations of curcumin, compared with the DMSO-control group (Fig. 2A). Furthermore, western blotting was used to detect the expression of apoptosis-related proteins. Fig. 2B revealed that curcumin significantly induced the downregulation of Bcl-2 and the upregulation of Bax expression in all of the three gastric cancer cell lines. Moreover, curcumin clearly cleaved pro-caspase- 3 and -9 to their active forms in each gastric cancer cell line at a concentration over $10 \mu \mathrm{M}$. Therefore, the aforementioned observations reveal that curcumin could induce apoptotic cell death in human gastric cancer cells in a dose-dependent manner.

Autophagy in gastric cancer cells is induced by curcumin. It has been reported that apoptosis and autophagy have a complex relationship in the process of cancer cell death $(23,24)$. Therefore, we wondered whether the activation of autophagy is also involved in the anticancer effect of curcumin. To confirm the ability of curcumin to trigger autophagy in human gastric cancer cells, we firstly performed AO staining to assess the formation of AVOs, a characteristic of autophagolysosomes, in curcumin-treated cancer cells. As shown in Fig. 3A, curcumin treatment resulted in pronounced accumulation of orange-red autophagic vacuoles in all of the three cell lines (BGC-823, 

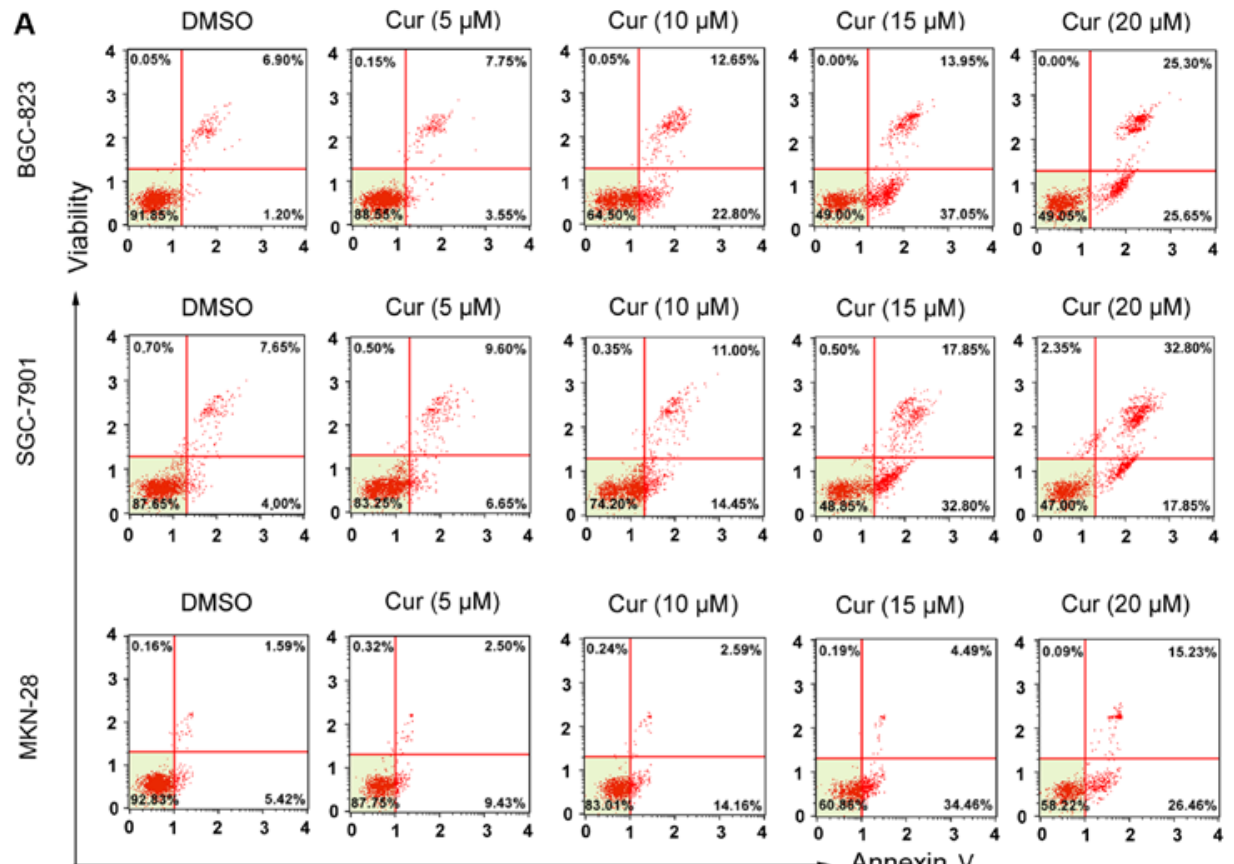

B
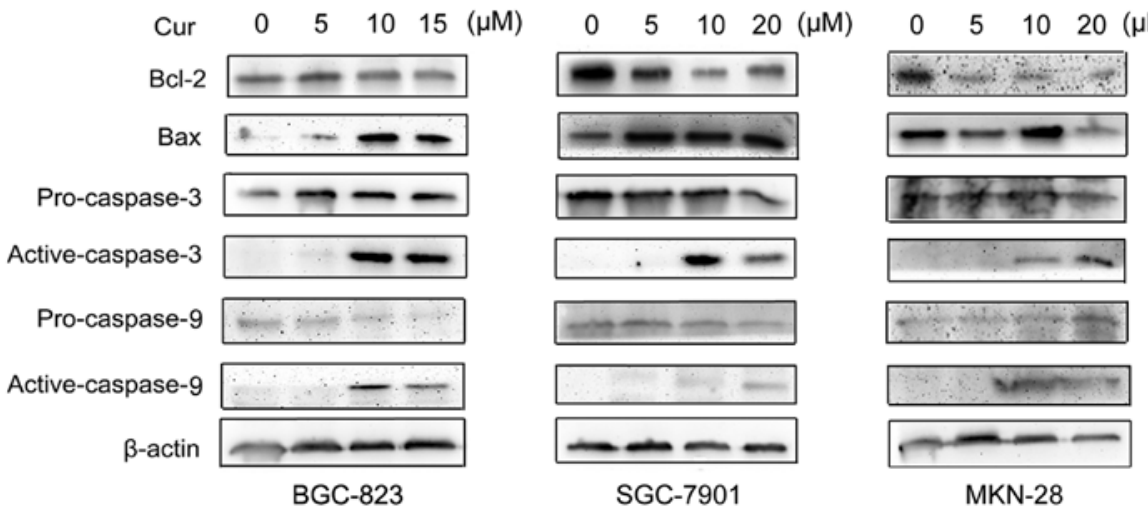

Figure 2. Apoptotic cell death in human gastric cancer cells confirmed by flow cytometry and western blotting after curcumin treatment. (A) BGC-823, SGC-7901 and MKN-28 cells were treated with curcumin at the indicated concentrations for $48 \mathrm{~h}$ and apoptosis was detected by flow cytometry with Annexin V staining. (B) After treatment with curcumin at the indicated concentrations for $48 \mathrm{~h}$, the expression of apoptosis-related proteins in gastric cancer cells was detected by western blotting. Cur, curcumin.

SGC-7901 and MKN-28) in a dose-dependent manner, whereas the DMSO-control group displayed green fluorescence, indicating the absence of AVO formation in the cytoplasm.

Furthermore, we detected conversion of microtubule-associated protein 1 light chain 3 (LC3) from LC3-I to LC3-II by western blotting assay. When autophagy is activated, LC3-I residing in the cytosol is cleaved to LC3-II and aggregated on the autophagosomal membranes. Transformation of LC3-I to LC3-II is thus a good marker for autophagy introduction. Our data showed LC3 turnover in all of the three different gastric cancer cell lines after exposure to curcumin and the accumulation of LC3-II was more significant following the increase of curcumin concentration (Fig. 3B). Thereafter, we investigated the regulatory effect of curcumin on the expression of autophagy-related (Atg) proteins in gastric cancer cells using western blotting assay. As shown in Fig. 3C, the levels of Beclin1, Atg7 and Atg5-Atg12-conjugate were all upregulated in BGC-823, SGC-7901 and MKN-28 cells by curcumin treatment in a dose-dependent manner, suggesting that curcumin has a potent ability to induce the activation of Atg protein expression in human gastric cancer cells, which is a critical characteristic for autophagy. Therefore, these data suggest that curcumin could induce autophagy activation as well as apoptotic cell death in human gastric cancer cells.

The PI3K/Akt/mTOR signaling pathway in gastric cancer cells is regulated by curcumin. Since the PI3K/Akt/mTOR signaling pathway has been confirmed to play an important role in the process of both apoptosis and autophagy, we further studied the effect of curcumin on the phosphorylation of Akt, mTOR and p70S6K in human gastric cancer cells. Results of western blotting assay conveyed that the expression levels of phospho-Akt in the three cell lines (BGC-823, SGC-7901 and MKN-28) were all obviously downregulated by curcumin treatment in a dose-dependent manner, followed by the downregulation of downstream phospho-mTOR and phospho-p70S6K (Fig. 4). This observation indicates that curcumin could inhibit the Akt/mTOR signaling pathway in gastric cancer cells, which may contribute to its induction of both apoptosis and autophagy in human gastric cancer cells. 

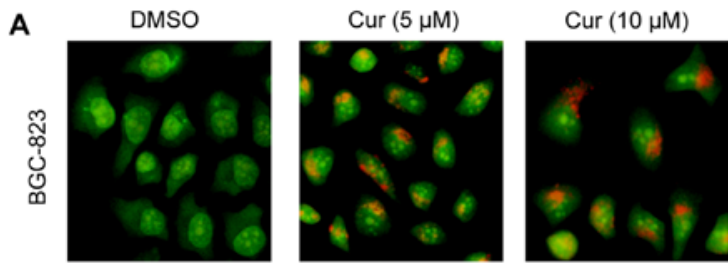

Cur $(15 \mu \mathrm{M})$

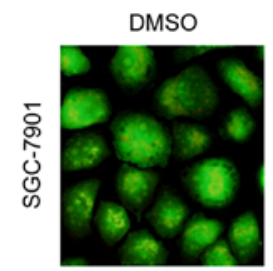

Cur $(5 \mu \mathrm{M})$

$\operatorname{Cur}(10 \mu \mathrm{M})$
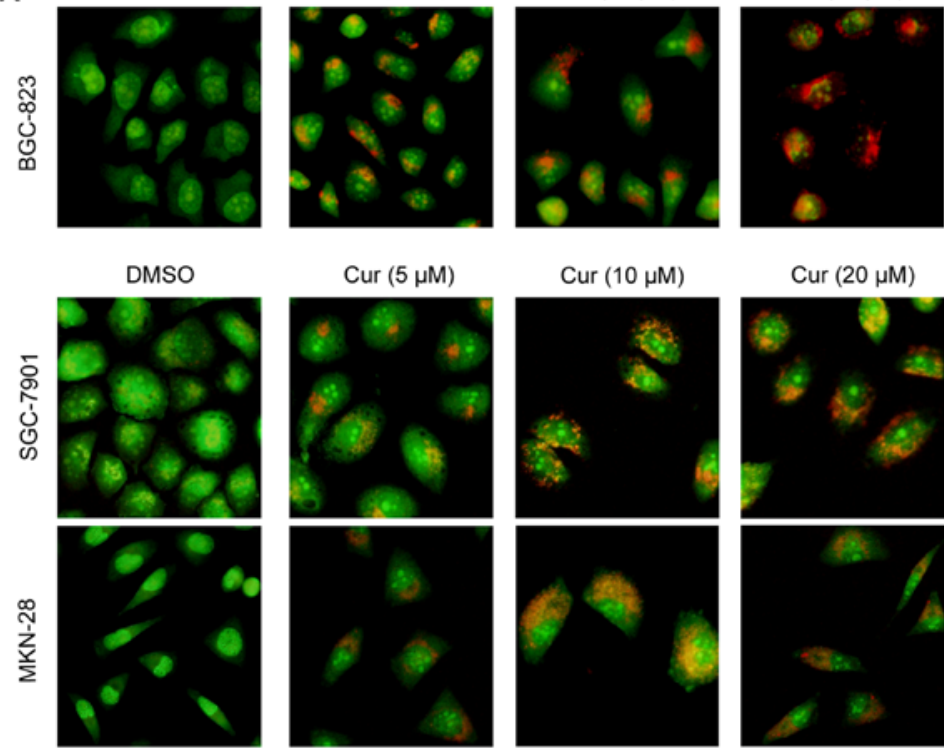

Cur $(20 \mu \mathrm{M})$
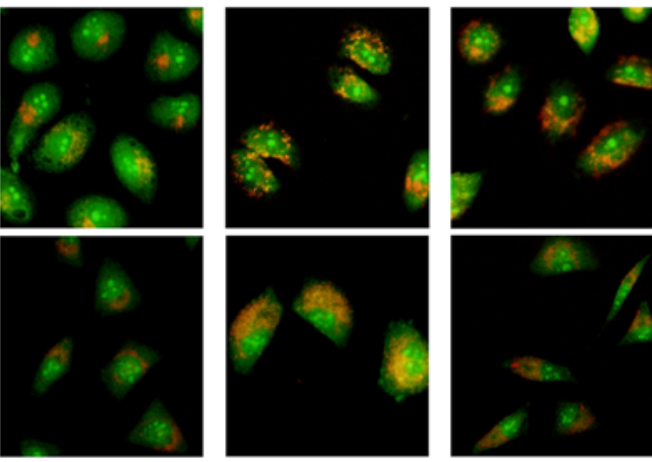

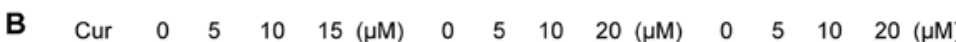

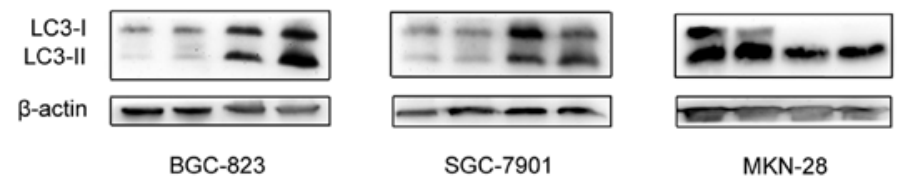

C Cur $\quad 0 \quad 5 \quad 10 \quad 15(\mu \mathrm{M})$
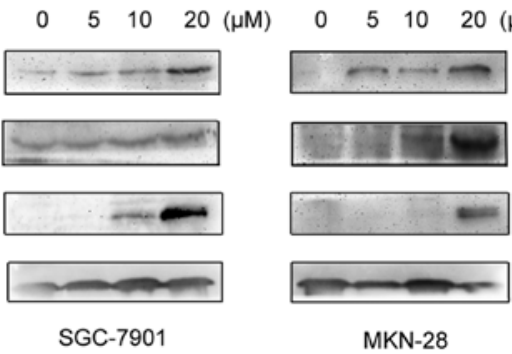

Figure 3. Activation of autophagy in human gastric cancer cells after curcumin treatment. (A) BGC-823, SGC-7901 and MKN-28 cells were treated with curcumin at the indicated concentrations for $48 \mathrm{~h}$ and the formation of acidic vesicular organelles (AVOs) was determined by acridine orange staining. (B) BGC823, SGC-7901 and MKN-28 cells were exposed to curcumin at the indicated concentrations for $48 \mathrm{~h}$ and the conversion of LC3-I to LC3-II was confirmed by western blotting assay. (C) After treatment with the indicated amounts of curcumin for 48 h, BGC-823, SGC-7901 and MKN-28 cells were assessed by western blotting for the expression of autophagy-related proteins. Cur, curcumin.
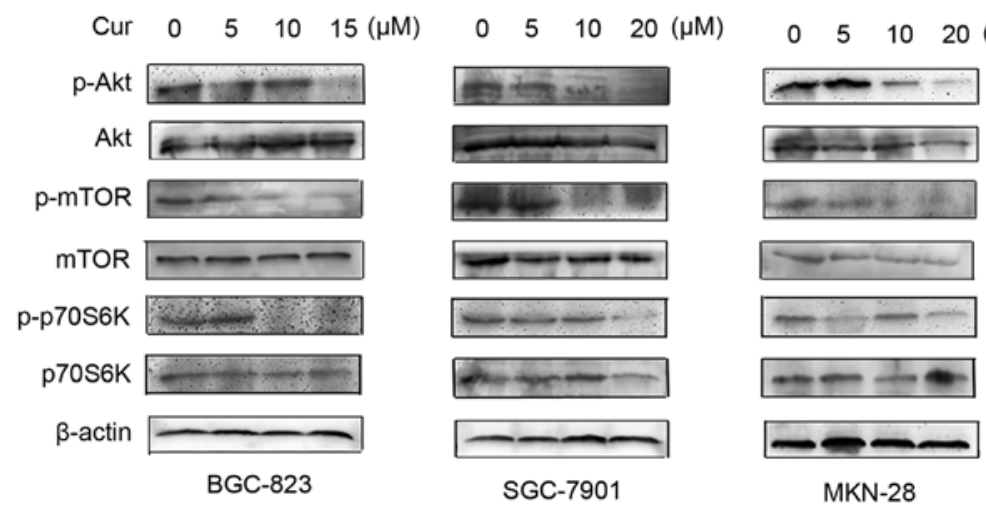

Figure 4. The PI3K/Akt/mTOR signaling pathway in human gastric cancer cells after curcumin treatment. Gastric cancer cells were treated with curcumin at the indicated concentrations for $48 \mathrm{~h}$ and the expression of phospho-Akt, phospho-mTOR and phospho-p70S6K was detected by western blotting. Cur, curcumin.

Proliferation and apoptotic cell death in gastric cancer cells after 3-MA treatment. Autophagy has been reported to have a paradoxical effect on cancer cell death or survival with various stimuli $(17,25)$. To study the contribution of autophagy 
A
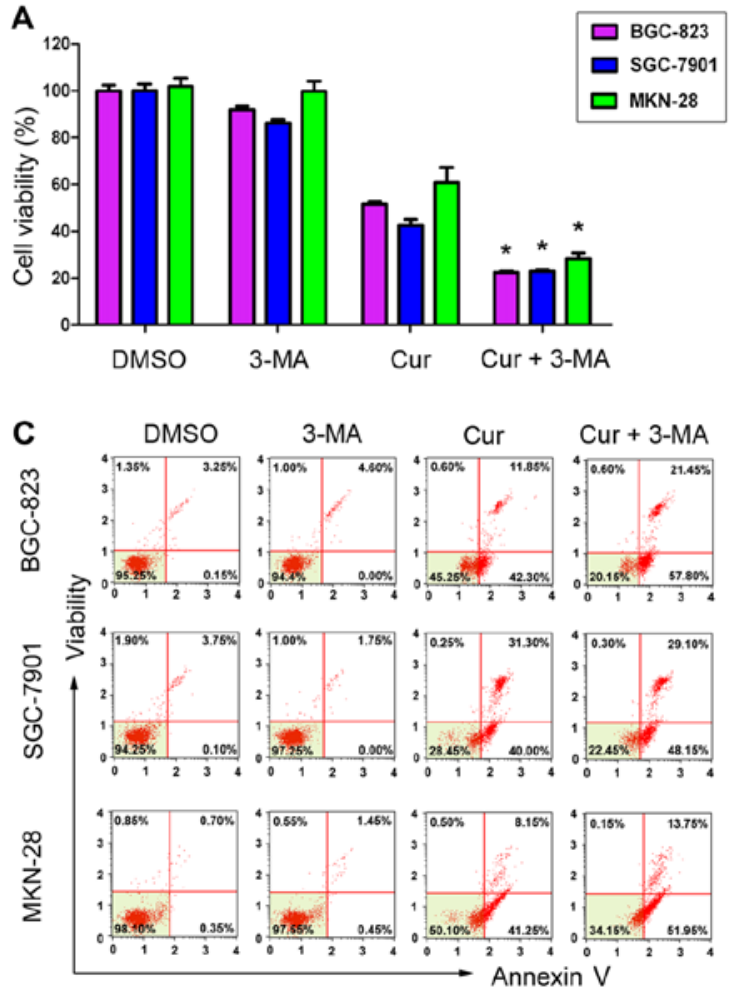

B
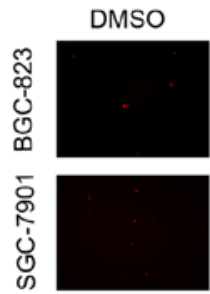

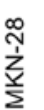

D
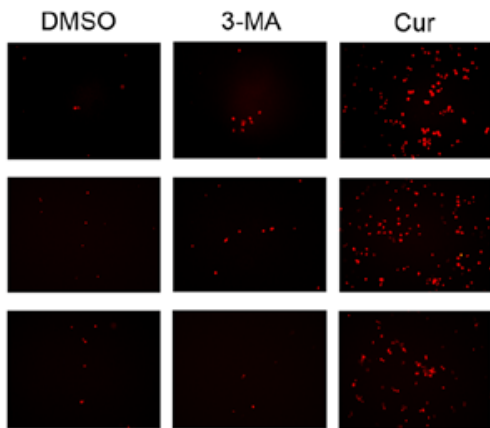

Cur + 3-MA
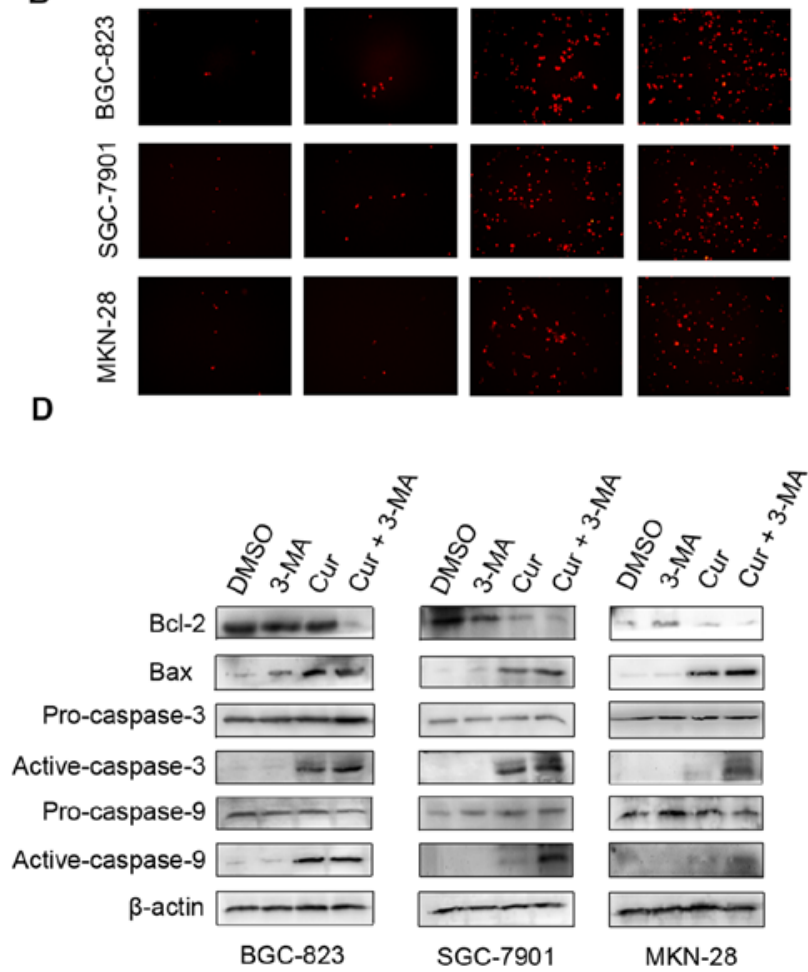

Figure 5. Viability and apoptosis in human gastric cancer cells after treatment with curcumin and autophagy inhibitor 3-MA. Gastric cancer cells were exposed to either curcumin (15 $\mu \mathrm{M}$ for BGC-823 cells and $20 \mu \mathrm{M}$ for SGC-7901 and MKN-28 cells) or 3-MA (10 mM), or a combination of curcumin and 3-MA for 48 h. (A) Cell viability was detected by MTT assay in gastric cancer cells. $\mathrm{P}<0.05$ vs. cells treated with curcumin alone. (B) Apoptotic cell death was assessed by TUNEL staining in gastric cancer cells (magnification, x200). (C) The apoptotic ratio was assessed by flow cytometry with Annexin V staining in gastric cancer cells. (D) The expression of apoptosis-related proteins was detected by western blotting in gastric cancer cells. Cur, curcumin.

to curcumin-induced apoptosis in human gastric cancer cells, we used the autophagy inhibitor 3-MA to prevent completion of autophagy at the early stage and co-treated the cancer cells with curcumin and 3-MA for $48 \mathrm{~h}$. As shown in Fig. 5A, curcumin and 3-MA co-treatment significantly decreased the viability of the three different gastric cancer cell lines, compared with the control group treated with curcumin alone. Furthermore, our data indicated that gastric cancer cells co-treated with curcumin and 3-MA exhibited increased apoptosis cell death compared with the control group treated with curcumin alone, which was demonstrated by a combination of TUNEL staining, flow cytometry and western blotting assays (Fig. 5B-D). Collectively, these results suggest that curcumin-induced autophagy could play a protective role in gastric cancer cell death and prevention of autophagy may enhance the anticancer effect of curcumin in human gastric cancer.

\section{Discussion}

Curcumin, a natural polyphenol derived from the root of turmeric (Curcuma longa), has been reported to be a potential traditional remedy in cancer prevention and therapy with pharmacological safety $(16,26)$. However, the effect of curcumin on human gastric cancer and the underlying mechanisms have not been well documented. Previous studies demonstrated that curcumin attenuates gastric cancer cell proliferation, induces apoptotic cell death and suppresses lymphatic vessel density in vivo or in vitro (27-29). In the present study, we provided the first evidence that curcumin inhibited cell growth and induced protective autophagy against apoptotic cell death in human gastric cancer.

As an anticancer agent, curcumin has been reported to play a potent role in the regulation of proliferation and apoptosis in various types of cancer. Bimonte et al revealed that curcumin treatment inhibited tumor growth and angiogenesis in human breast cancer both in vivo and in vitro (14). Koprowski et al revealed that curcumin could induce growth suppression and apoptosis of cholangiocarcinoma at low treatment concentrations (30). Zou et al reported that an analog of curcumin, WZ35, exhibited the anticancer effect on gastric cancer via activation of the JNK and ER stress apoptotic pathways mediated by ROS generation (31).

In the present study, proliferation was obviously inhibited in both a time- and dose-dependent manner in three different gastric cancer cell lines (BGC-823, SGC-7901 and MKN-28) treated with curcumin. Moreover, curcumin was demonstrated to induce marked apoptosis in human gastric cancer cells in a dose-dependent manner, which was conveyed by the increase in TUNEL-positive cells and the apoptosis population by flow cytometry, as well as the upregulation of apoptosis-related proteins. Consistent with our findings, studies have majorly focused on apoptosis to elucidate the mechanisms by which curcumin exerts its anticancer effect $(16,28)$. However, the role of curcumin-induced autophagy and the exact mechanism involved in the interaction between apoptosis and autophagy with curcumin treatment have not been generally clarified, particularly in human gastric cancer. 
Autophagy, a catabolic degradation process in which cellular proteins or organelles are degraded in the lysosome and recycled, is designated as a non-apoptotic form of programmed cell death called autophagy-induced cell death. It has recently emerged as a critical player in the development of different diseases, particularly cancer. Accumulating studies have reported that medicinal plant-derived compounds or extracts with anticancer properties, such as curcumin, could induce autophagy in various types of cancer and it has been established that the role of autophagy in human cancer is complicated. Kim et al revealed that curcumin-induced autophagy acts as a pro-death signal, which contributes to the decreased survival of oral cancer cells (32). In contrast, Zhou et al demonstrated that a novel curcumin analog EF25-(GSH) $)_{2}$ which induced autophagy could be inhibited by chloroquine, thus leading to significantly enhanced apoptosis and cytotoxicity in hepatocellular carcinoma (33). Moreover, Wang et al revealed that quercetin could induce autophagy antagonizing apoptotic cell death in gastric cancer cells, by modulation of the Akt/mTOR and HIF-1 $\alpha$ signaling pathways (20).

In the present study, curcumin treatment caused a dosedependent formation of acidic vesicular organelles (AVOs) in human gastric cancer cells. Western blotting also demonstrated a conversion from LC3-I to LC3-II and the upregulated expression of autophagy-related proteins including Beclin1, Atg7 and Atg5-Atg12 conjugate in gastric cancer cells exposed to curcumin. These characteristic changes firstly demonstrated that curcumin could trigger the autophagic process in human gastric cancer cells in vitro. Furthermore, whether curcumin-induced autophagy plays a protective role or promotes cell death in gastric cancer was confirmed later in our investigation.

The PI3K/Akt/mTOR signaling pathway has been reported to be the major negative regulator of both apoptosis and autophagy. Numerous signaling molecules of the $\mathrm{PI} 3 \mathrm{~K} / \mathrm{Akt} / \mathrm{mTOR}$ pathway have oncogenic properties and constitutive activation of this signaling pathway is often involved in the progression of various human cancers $(34,35)$. Contrary to tumor suppressor genes such as PTEN and p53 which can stimulate autophagy, oncogenes of PI3K and Akt have been confirmed to play an inhibiting role in autophagy activation. The results of our present study revealed that curcumin treatment obviously inhibited the phosphorylation of Akt and downstream mTOR as well as p70S6K in all of the three different human gastric cancer cell lines in vitro. This finding indicated that the inhibition of the PI3K/Akt/mTOR signaling pathway may play an important role in the autophagy of gastric cancer cells treated with curcumin. However, since other signaling pathways such as Erk1/2, AMPK and JNK may be also responsible for autophagy activation, further research is still warranted in the future.

In addition, autophagy inhibitor 3-MA was used in the present study, to prevent autophagy in order to further investigate the dual role of curcumin-induced autophagy in gastric cancer cells. Consequently, our results demonstrated that autophagy inhibition significantly decreased cell viability and enhanced apoptotic cell death in human gastric cancer cells treated with curcumin. These findings revealed that the prevention of autophagy significantly promoted the anticancer effect and the toxicity of curcumin in gastric cancer. In other words, curcumin-induced autophagy plays a protective role in human gastric cancer, which may lead to drug resistance by curcumin in cancer treatment. Therefore, inhibition of this protective autophagy with the application of an autophagy inhibitor may improve the therapeutic efficacy of curcumin in human gastric cancer.

In summary, the present study demonstrated for the first time that the anticancer effect of curcumin was associated with its inhibition of proliferation and promotion of apoptotic cell death in gastric cancer cells. Moreover, the present study provides the first evidence that curcumin induced a protective autophagy antagonizing apoptotic cell death in human gastric cancer cells and combination of curcumin with an autophagy inhibitor could enhance the anticancer toxicity of this chemotherapeutic drug. Thus, it can be concluded that our findings provide a valuable strategy for improving therapeutic treatment with curcumin or its analog in human gastric cancer.

\section{Acknowledgements}

The present study was supported by the National Natural Science Foundation of China (grant no. 81402280), the Foundation from Jiangsu Key Laboratory of Medical Science and Laboratory Medicine (JSKLM-2014-005), and the Doctor Foundation from the First People's Hospital of Lianyungang (grant no. BS1503).

\section{References}

1. Kim MJ and Kim H: Anticancer effect of lycopene in gastric carcinogenesis. J Cancer Prev 20: 92-96, 2015.

2. Jeong SH, Kim YW, Yu W, Lee SH, Park YK, Park SH, Jeong IH Lee SE, Park Y and Lee YJ: High morbidity in myocardial infarction and heart failure patients after gastric cancer surgery. World J Gastroenterol 21: 6631-6638, 2015.

3. Ford $\mathrm{H}$ and Gounaris I: Docetaxel and its potential in the treatment of refractory esophagogastric adenocarcinoma. Therap Adv Gastroenterol 8: 189-205, 2015.

4. Du W, Li C, Wang H, Zhao A, Shen J, Yong F and Jia H: Effect of neoadjuvant chemotherapy on sevoflurane MAC-BAR value of patients undergoing radical stomach carcinoma surgery. Int $\mathrm{J}$ Clin Exp Med 8: 5649-5657, 2015.

5. Alizadeh AM, Sadeghizadeh M, Najafi F, Ardestani SK, Erfani-Moghadam V, Khaniki M, Rezaei A, Zamani M, Khodayari S, Khodayari H, et al: Encapsulation of curcumin in diblock copolymer micelles for cancer therapy. Biomed Res Int 824746: 2015, 2015.

6. Chang R, Sun L and Webster TJ: Short communication: Selective cytotoxicity of curcumin on osteosarcoma cells compared to healthy osteoblasts. Int J Nanomedicine 9: 461-465, 2014.

7. Zanotto-Filho A, Braganhol E, Klafke K, Figueiró F, Terra SR, Paludo FJ, Morrone M, Bristot IJ, Battastini AM, Forcelini CM, et al: Autophagy inhibition improves the efficacy of curcumin/temozolomide combination therapy in glioblastomas. Cancer Lett 358: 220-231, 2015.

8. Marquardt JU, Gomez-Quiroz L, Arreguin Camacho LO, Pinna F, Lee YH, Kitade M, Domínguez MP, Castven D, Breuhahn K, Conner EA, et al: Curcumin effectively inhibits oncogenic NF- $\mathrm{KB}$ signaling and restrains stemness features in liver cancer. J Hepatol 63: 661-669, 2015.

9. Shakibaei M, Kraehe P, Popper B, Shayan P, Goel A and Buhrmann C: Curcumin potentiates antitumor activity of 5-fluorouracil in a 3D alginate tumor microenvironment of colorectal cancer. BMC Cancer 15: 250, 2015.

10. Wu J, Cai Z, Wei X, Chen M, Ying S, Shi L, Xu RA, He F, Liang $\mathrm{G}$ and Zhang X: Anti-lung cancer activity of the curcumin analog JZ534 in vitro. Biomed Res Int 2015: 504529, 2015.

11. Terlikowska KM, Witkowska AM, Zujko ME, Dobrzycka B and Terlikowski SJ: Potential application of curcumin and its analogues in the treatment strategy of patients with primary epithelial ovarian cancer. Int J Mol Sci 15: 21703-21722, 2014. 
12. Coleman DT, Soung YH, Surh YJ, Cardelli JA and Chung J: Curcumin prevents palmitoylation of integrin $\beta 4$ in breast cancer cells. PLoS One 10: e0125399, 2015.

13. Mishra A, Kumar R, Tyagi A, Kohaar I, Hedau S, Bharti AC, Sarker S, Dey D, Saluja D and Das B: Curcumin modulates cellular AP-1, NF-kB, and HPV16 E6 proteins in oral cancer. Ecancermedicalscience 9: 525, 2015.

14. Bimonte S, Barbieri A, Palma G, Rea D, Luciano A, D'Aiuto M, Arra C and Izzo F: Dissecting the role of curcumin in tumour growth and angiogenesis in mouse model of human breast cancer. Biomed Res Int 878134: 2015, 2015.

15. Wu J, Lu WY and Cui LL: Inhibitory effect of curcumin on invasion of skin squamous cell carcinoma A431 cells. Asian Pac J Cancer Prev 16: 2813-2818, 2015.

16. Amin AR, Haque A, Rahman MA, Chen ZG, Khuri FR and Shin DM: Curcumin induces apoptosis of upper aerodigestive tract cancer cells by targeting multiple pathways. PLoS One 10: e0124218, 2015.

17. Zhi X and Zhong Q: Autophagy in cancer. F1000Prime Rep 7: $18,2015$.

18. Galluzzi L, Pietrocola F, Bravo-San Pedro JM, Amaravadi RK, Baehrecke EH, Cecconi F, Codogno P, Debnath J, Gewirtz DA, Karantza V, et al: Autophagy in malignant transformation and cancer progression. EMBO J 34: 856-880, 2015.

19. Zarzynska JM: The importance of autophagy regulation in breast cancer development and treatment. Biomed Res Int 2014: 710345, 2014.

20. Wang K, Liu R, Li J, Mao J, Lei Y, Wu J, Zeng J, Zhang T, Wu H, Chen L, et al: Quercetin induces protective autophagy in gastric cancer cells: Involvement of Akt-mTOR- and hypoxia-induced factor $1 \alpha$-mediated signaling. Autophagy 7: 966-978, 2011

21. Lin Y, Wang K, Hu C, Lin L, Qin S and Cai X: Elemene injection induced autophagy protects human hepatoma cancer cells from starvation and undergoing apoptosis. Evid Based Complement Alternat Med 2014: 637528, 2014

22. Zhang SF, Wang XL, Yang XQ and Chen N: Autophagyassociated targeting pathways of natural products during cancer treatment. Asian Pac J Cancer Prev 15: 10557-10563, 2014.

23. Zhang MS, Niu FW and Li K: Proflavin suppresses the growth of human osteosarcoma MG63 cells through apoptosis and autophagy. Oncol Lett 10: 463-468, 2015.

24. Liu D, Gao M, Yang Y, Qi YU, Wu K and Zhao S: Inhibition of autophagy promotes cell apoptosis induced by the proteasome inhibitor MG-132 in human esophageal squamous cell carcinoma EC9706 cells. Oncol Lett 9: 2278-2282, 2015.
25. Wang X, Qi W, Li Y, Zhang N, Dong L, Sun M, Cun J, Zhang Y, Lv S and Yang Q: Huaier extract induces autophagic cell death by inhibiting the mTOR/S6K pathway in breast cancer cells. PLoS One 10: e0131771, 2015.

26. Rahmani AH, AlZohairy MA, Aly SM and Khan MA: Curcumin: A potential candidate in prevention of cancer via modulation of molecular pathways. Biomed Res Int 2014: 761608, 2014.

27. Liu X, Sun K, Song A, Zhang X, Zhang X, He X and Xiaodong H: Curcumin inhibits proliferation of gastric cancer cells by impairing ATP-sensitive potassium channel opening. World J Surg Oncol 12: 389, 2014

28. Liang T, Zhang X, Xue W, Zhao S, Zhang X and Pei J: Curcumin induced human gastric cancer BGC-823 cells apoptosis by ROS-mediated ASK1-MKK4-JNK stress signaling pathway. Int J Mol Sci 15: 15754-15765, 2014.

29. Da W, Zhu J, Wang L and Sun Q: Curcumin suppresses lymphatic vessel density in an in vivo human gastric cancer model. Tumour Biol 36: 5215-5223, 2015

30. Koprowski S, Sokolowski K, Kunnimalaiyaan S, Gamblin TC and Kunnimalaiyaan M: Curcumin-mediated regulation of Notch1/hairy and enhancer of split-1/survivin: Molecular targeting in cholangiocarcinoma. J Surg Res 198: 434-440, 2015.

31. Zou P, Zhang J, Xia Y, Kanchana K, Guo G, Chen W, Huang Y, Wang Z, Yang S and Liang G: ROS generation mediates the anti-cancer effects of WZ35 via activating JNK and ER stress apoptotic pathways in gastric cancer. Oncotarget 6: 5860-5876, 2015.

32. Kim JY, Cho TJ, Woo BH, Choi KU, Lee CH, Ryu MH and Park HR: Curcumin-induced autophagy contributes to the decreased survival of oral cancer cells. Arch Oral Biol 57: 1018-1025, 2012.

33. Zhou T, Ye L, Bai Y, Sun A, Cox B, Liu D, Li Y, Liotta D, Snyder JP, Fu H, et al: Autophagy and apoptosis in hepatocellular carcinoma induced by EF25-(GSH) : A novel curcumin analog. PLoS One 9: e107876, 2014.

34. Yip PY: Phosphatidylinositol 3-kinase-AKT-mammalian target of rapamycin (PI3K-Akt-mTOR) signaling pathway in non-small cell lung cancer. Transl Lung Cancer Res 4: 165-176, 2015.

35. Cheaib B, Auguste A and Leary A: The PI3K/Akt/mTOR pathway in ovarian cancer: Therapeutic opportunities and challenges. Chin J Cancer 34: 4-16, 2015. 